\title{
A Population of Linear, Second Order, Elliptic Partial Differential Equations on Rectangular Domains. Part I*
}

\author{
By John R. Rice, Elias N. Houstis and Wayne R. Dyksen
}

\begin{abstract}
We present a population of 56 linear, two-dimensional elliptic partial differential equations (PDEs) suitable for evaluating numerical methods and software. Forty-two of the PDEs are parametrized which allows much larger populations to be made; 189 specific cases are presented here along with solutions (some are only approximate). Many of the PDEs are artificially created so as to exhibit various mathematical behaviors of interest; the others are taken from "real world" problems in various ways. The population has been structured by introducing measures of complexity of the operator, boundary conditions, solution and problem. The PDEs are first presented in mathematical terms along with contour plots of the 189 specific solutions. Machine-readable descriptions are given in Part 2; many of the PDEs involve lengthy expressions and about a dozen involve extensive tabulations of approximate solutions.
\end{abstract}

\section{Introduction}

2. Characteristics of the problem

\subsection{Sources}

\subsection{Problem Features and Complexity Classifications}

\section{Format of Problem Descriptions}

\section{Appendix 1: Tabulations of Population Characteristics}

Appendix 2: Mathematical Descriptions and Solution Contours (microfiche supplement)

Part 2: Machine-Readable Descriptions (microfiche supplement)

1. Introduction. The motivation for creating this PDE population is for use in the evaluation of numerical methods and PDE software. The need and rationale for a systematic approach to such evaluations is given in Rice [10], Houstis and Rice [8], Crowder, Dembo and Mulvey [2]; it suffices here to say that a properly chosen problem population is an essential ingredient for a sound evaluation of numerical methods and software.

A useful population of PDEs is inevitably very lengthy and this one is no exception as one sees from the last two appendices. Thus in the body of this paper we discuss the sources of the PDEs, how they are described in the appendices and

Received July 29, 1980.

1980 Mathematics Subject Classification. Primary 65N99.

Key words and phrases. Elliptic partial differential equations, numerical methods, software evaluation, population of problems, linear, second order.

*Sponsored by the United States Army under contract Nos. DAAG29-75-C-0024 and DAAG29-80C-0041. This material is based upon work supported by the National Science Foundation under Grant Nos. MCS77-01408 and MCS79-01437. 
how a structure has been created in the population through the use of quantitative (but subjective) measures of features.

It is important that one be able to create relevant subpopulations as one inevitably wants to evaluate methods for particular subclasses of PDEs (e.g., separable, with singularities or with mixed boundary conditions). Experience shows that no one universal method is best for all PDEs (even in this rather restricted context) and one of the important tasks of research is to create and/or identify methods that are especially efficient for particular classes of PDEs. Once one embarks on such a task one sees that this population, which originally might seem large and bulky, is actually rather small for the uses to be made of it. It is only the fact that it can be substantially expanded in various directions through the parametrization that gives one hope that it is adequate for a wide variety of evaluations.

2. Characteristics of the Problems. A source parameter is assigned to each PDE which ranges from 0 (artificial problem) to 100 (actual real world problem). This feature, as the others introduced later, is subjective in nature and the values given must be taken as approximate indications of our intuitive feelings. The PDE $u_{x x}+u_{y y}=1$ might be completely artificial for one person and be the actual applications PDE for another. We have at least tried to be consistent in these values.

2.1. Sources. Many problems have been normalized so the maximum value of the solution is 1.0 and almost all have this value between .1 and 100. Many of the domains have been standardized to the unit square, $0<x, y<1$. The sources of the PDEs are:

A. Problems used in previous studies. Nine problems are included which were used by Eisenstat and Schultz [3] or Houstis et al. [4] and [5]. Subsets of this populaton have been used by Houstis and Papatheodorou [6] and [7] and Lynch and Rice [9]. Some of these PDEs have had parameters added and all have been normalized so the maximum value of the solution is about 1.0.

B. Artificial problems. Many problems have been created just to exhibit various mathematical behaviors of interest (e.g. singularities, oscillations or wave fronts). Such behaviors are important for theory or application (or both) and one needs to have them present in the population in an easily identifiable manner.

C. Problems adapted from the "real world". A persistent difficulty is the desire to have PDEs which represent the "real world" and the necessity to know their true solutions. Among the strategies to adapt real world problems we have used:

(i) choosing explicit functions which model the physical solutions and then determining appropriate boundary conditions and/or right side to make this the true solution;

(ii) using truncated series expansions (of high accuracy) with appropriate small modifications in the boundary conditions or right side;

(iii) solving nonlinear problems approximately, then substituting the tabulated numerical solution into the operator (using quadratic interpolation from a 10 by 10 grid) to obtain a linear problem which is, in turn, solved approximately. In these cases the true solution is not known, but the machine-readable population contains tabulated values of a hopefully accurate numerical solution. 
2.2. Problem Features and Complexity Classifications. We identify as problem features the smoothness and local variation of operator, the boundary conditions and the solution. These features are quantified on a one-dimensional scale of 0 to 100 even though there are rather independent properties that can be called smoothness or local variation. These features are measured subjectively from the following descriptions of the scale.

Smoothness. This refers to the mathematical properties of the functions or operators involved. Key points on the scale are

$00=$ entire functions or constants,

$10=$ analytic; very well behaved,

30 = very smooth, some higher derivative ( 5 or so) discontinuity possible,

$50=$ still smooth, third derivative discontinuity possible,

$70=$ not rough to the eye, but possibly only 1 continuous derivative,

$80=$ continuous, functions might be theoretically smooth but rough on a gross scale,

$90=$ possibly discontinuous, nearly singular functions or operators,

$100=$ strong singularities like $1 / x$ or $1 / x^{2}$.

Local variation. This refers to how much a function changes (relative to its size) in a small part of its domain. These variations might be oscillations, wave fronts, peaks or boundary layers. Key points on the scale are

$00=$ very smooth, uniform,

$10=$ mild variation, probably convex, some nonuniformity, e.g. $\sin (2 x), e^{3 x}$ on $[0,1]$

$25=$ modest variation of oscillation; mild wave front or peak, e.g. $\sin (6 x)$, $1 /\left(1+100 x^{4}\right)$ on $[0,1]$,

$40=$ considerable peak or oscillation; change of magnitude occurs within 10 $15 \%$ of domain,

60 = sharp peaks, wave fronts, boundary layers or oscillations; $100 \%$ change in magnitude occurs within $5 \%$ of domain,

75 = practically a discontinuity in magnitude; continuity observable only with a fine scale examination,

90 = actual discontinuity in magnitude; extreme oscillation, step functions, e.g. $\sin (300 x)$ on $[0,1]$.

The overall problem complexity is represented by the average of the above six feature measures. The problems in this population do not have complexities exceeding 58 (only one exceeds 50), a level which might be interpreted as "rather messy with one or two substantial complications". The problem feature measures are included in the descriptions along with the source parameter.

Appendix 1 presents some summary information about the population. Tables are given which

A. group the PDEs according to types of the operator and boundary conditions (e.g. Helmholtz and Dirichlet or constant coefficients and mixed),

B. list the 56 PDEs with abbreviated feature descriptions,

C. group the PDEs according to the smoothness of the operator and right side,

D. group the PDEs according to the smoothness of the solution. 
3. Format of Problem Descriptions. Appendix 2 contains a mathematical description of each PDE along with contour plots for each specific instance included in the set of 189 PDEs. An example is shown in Figure 1. The description begins with a problem number and source followed by a mathematical description of the PDE. Then brief comments are given for the operator, right side, boundary conditions, solution and parameters (if any). Sometimes functions appearing in the mathematical description are defined in these comments.

Four generic functions are used:

$f(x, y)=$ right side of PDE determined so that the given true solution is correct.

$f(x), g(y)=$ right sides of boundary conditions determined so that the given true solution is correct.

$T(x, y)=$ the true solution, used in the coefficients of some PDEs derived from nonlinear problems.

$r(x, y)=$ an approximate solution used in some PDEs whose true solution is unknown.

Contour plots are given for one or more particular PDEs for each problem. The border of the plots contains the following information:

(i) values of the parameters, the variables $A, B, C$, etc. denote $\alpha, \beta$, $\gamma$, etc.

(ii) maximum and minimum values of the solution; the contours are equispaced between these values.

(iii) the classification parameters in the form

$$
\text { S.P O1.O2 B1.B2 S1.S2 }
$$

where

$$
\begin{array}{ll}
S=\text { source parameter } & P=\text { problem complexity } \\
\alpha 1=\text { smoothness feature } & \alpha 2=\text { local variation feature } \\
\text { and } \alpha=O \text { for the operator, } & \mathrm{B} \text { for the boundary conditions, }
\end{array}
$$

$\mathrm{S}$ for the solution.

The machine-readable description of the PDE population consists of two files: EQNFIL and MACFIL. EQNFIL has 189 entries which are either complete statements of the PDE in the ELLPACK language (see Boisvert, Houstis and Rice [1]) or a reference to an entry in MACFIL with values given for parameters. See Figure 2 for a short example. The information given starts with the problem number, feature parameter values and a code for various attributes of the PDE which are used within the ELLPACK system. The machine-readable description uses $\mathrm{A}, \mathrm{B}, \mathrm{C}$, etc. for $\alpha, \beta, \gamma$, etc. Then ELLPACK language code is given for the operator and boundary conditions; this code should be self explanatory once one sees the UXX\$ represents $u_{x x}$, etc. Finally, there is a Fortran code for any functions that appear in the operator, right side or boundary conditions. This latter code averages about 20 lines and can be as much as 150 lines (excluding tables that are part of some problems). These descriptions are given in Part 2 of this report.

MACFIL entries are just like EQNFIL descriptions of a PDE except that the places where parameter values are to be substituted are indicated by $\& A, \& B$, etc. $A$ refers to the first parameter, $B$ the second and so on. There are somewhat more than 8500 lines in these two files. 

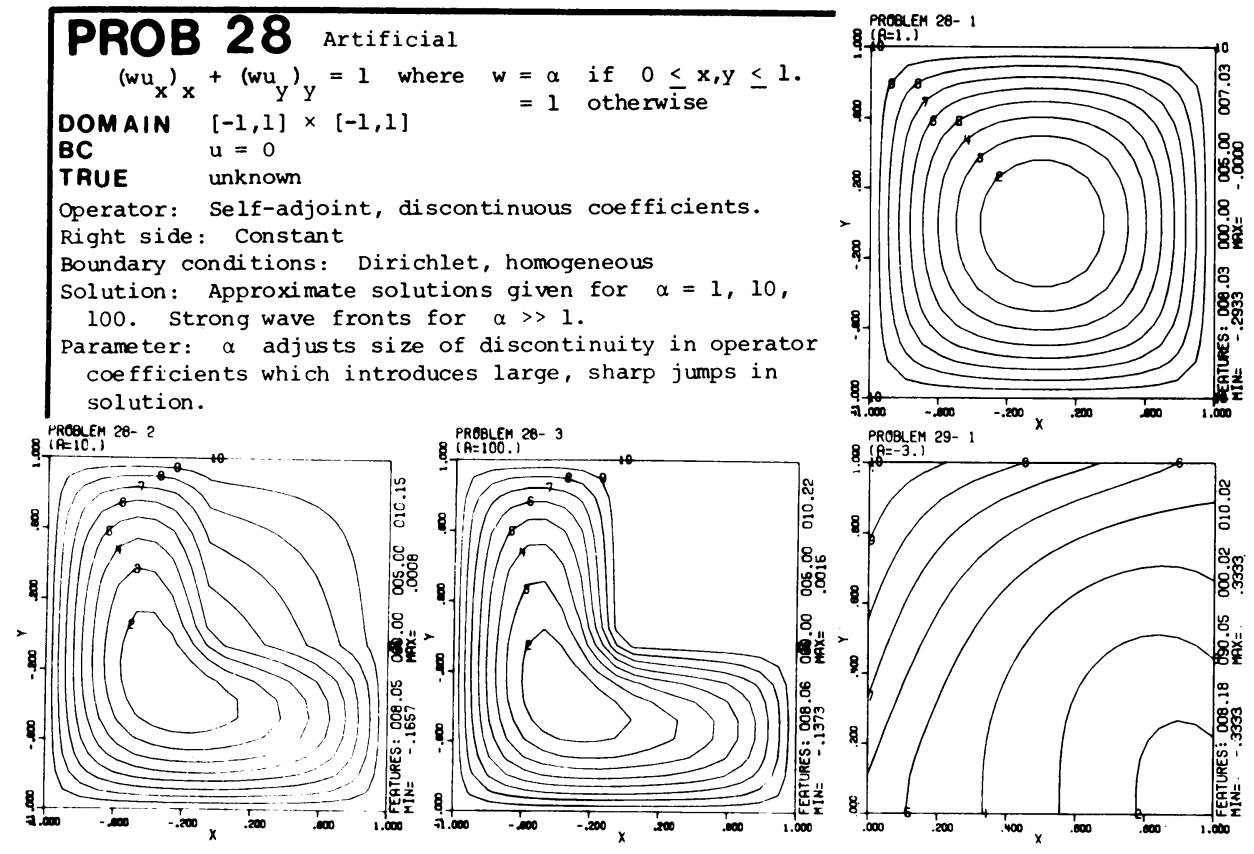

FIGURE 1

An example of the mathematical description of a PDE along

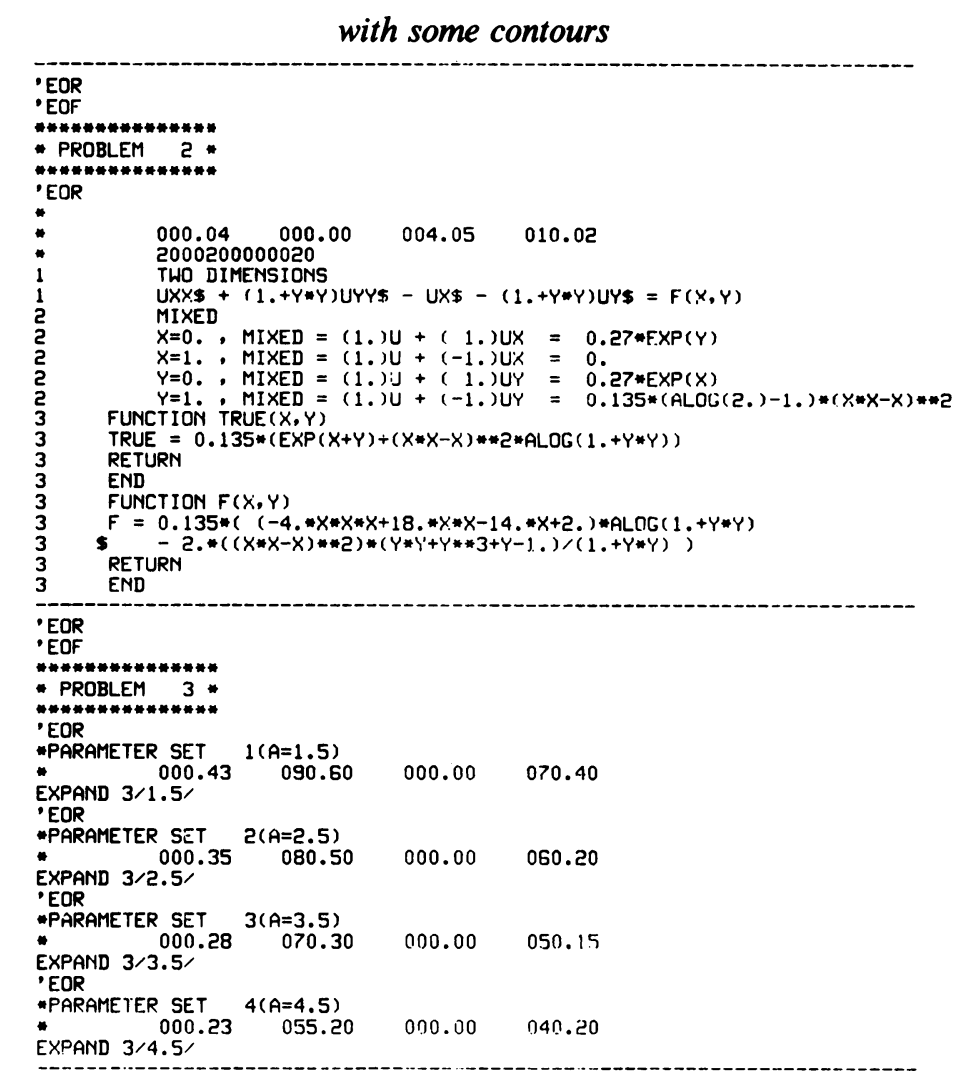

FIGURE 2

A sample from EQNFIL showing a short PDE description in machine-readable form and a reference to a similar description in MACFIL 
Acknowledgements. This work has been supported in part as follows: National Science Foundation grant MCS77-01408 (Rice, Houstis and Dyksen), United States Army Contract DAAG29-75-C-0024 (Rice) and National Science Foundation grant MCS79-01437 (Houstis).

Mathematics Research Center

University of Wisconsin

Madison, Wisconsin 53706

Department of Mathematics and Computer Science

University of South Carolina

Columbia, South Carolina 29208

Department of Mathematics

Purdue University

West Lafayette, Indiana 47907

1. R. E. Boisvert, E. N. Houstis \& J. R. Rice, “A system for performance evaluation of partial differential equations software," IEEE Trans. Software Engrg., v. 5, 1979, pp. 418-425.

2. H. Crowder, R. S. Dembo \& J. M. Mulvey, "On reporting computational experiments with mathematical software," ACM Trans. Math. Software, v. 5, 1979, pp. 191-203.

3. S. C. Eisenstat \& M. H. Schultz, "Computational aspects of the finite element method," Complexity of Sequential and Parallel Algorithms (J. F. Traub, Ed.), Academic Press, New York, 1973, pp. 271-282.

4. E. N. Houstis, R. E. Lynch, T. S. Papatheodorou \& J. R. Rice, "Development, evaluation and selection of methods for elliptic partial differential equations," Ann. Assoc. Calcul. Analog., v. 11, 1975, pp. 98-105.

5. E. N. Houstis, R. E. Lynch, T. S. Papatheodorou \& J. R. Rice, "Evaluation of numerical methods for elliptic partial differential equations," J. Comput. Phys., v. 27, 1978, pp. 323-350.

6. E. N. Houstis \& T. S. PAPATHEOdorou, "Comparison of fast direct methods for elliptic problems," Advances in Computer Methods for Partial Differential Equations II (R. Vishnevetsky, Ed.) IMACS, Rutgers University, New Brunswick, N.J., 1977, pp. 46-52.

7. E. N. Houstis \& T. S. PAPATHEOdorou, "High order fast elliptic solver," ACM Trans. Math. Software, v. 5, 1979, pp. 431-441.

8. E. N. Houstis \& J. R. RICE, "An experimental design for the computational evaluation of elliptic partial differential equation solvers," The Production and Assessment of Numerical Software (M. A. Hennell, Ed.), Academic Press, New York, 1980.

9. R. E. LYNCH \& J. R. RICE, "The Hodie method and its performance," Recent Advances in Numerical Analysis (C. de Boor, Ed.), Academic Press, New York, 1978, pp. 143-179.

10. J. R. RICE, "Methodology for the algorithm selection problem," Performance Evaluation of Numerical Software (L. D. Fosdick, Ed.), North-Holland, Amsterdam, 1979, pp. 301-307. 
PARTIAL DIFFERENTIAL EQUATIONS

\section{Appendix 1: Tabulations of Population Characteristics}

\begin{tabular}{|c|c|c|c|c|c|c|}
\hline \multicolumn{7}{|c|}{$\begin{array}{l}\text { Table } 1 \\
\text { cations of Problems } \\
\text { ator and Boundary Conditions }\end{array}$} \\
\hline \multirow{2}{*}{ Operator } & \multicolumn{3}{|c|}{ Constant Cocflicients } & \multicolumn{3}{|c|}{ Non-Constant Coefficients } \\
\hline & Dirichlet & Ncumann & Mixed & Dirichlet & Neumann & Mixed \\
\hline Laplace & $\begin{array}{r}3, \quad 7,7, \\
8,10,11, \\
17,33,34 \\
35,47,50\end{array}$ & & $\begin{array}{l}4,31,35 \\
38,55\end{array}$ & & & \\
\hline $\begin{array}{l}\text { Helmholtz } \\
\text { Type }\end{array}$ & $9,41,53$ & & & $\begin{array}{r}6,20,39 \\
44,45,48 \\
49\end{array}$ & & \\
\hline Self-Adjoint & 5 & & & $\begin{array}{r}1,13,22 \\
25,28,54\end{array}$ & & $\begin{array}{l}1,19,23, \\
52\end{array}$ \\
\hline General & 14,46 & 42 & 43 & $\begin{array}{l}12,15,16, \\
18,21,26, \\
27,29,30 \\
32,36,37 \\
56\end{array}$ & 24 & $\begin{array}{l}2,23,24, \\
40,51\end{array}$ \\
\hline
\end{tabular}

Note that problems 1,4 , and 35 appear in two places in the table since they have boundary conditions of the form $u+\alpha u_{N}=g$ and hence have Dirichlet boundary conditions for $\alpha=0$. Problem 24 appears in two places since it has boundary conditions of the form $\beta u+u_{x}+u_{y}=0$ and is Neumann for $\beta=0$. 
Table 2

\section{Problem Characteristics}

The principal characteristics are tabulated below using the following encodings:

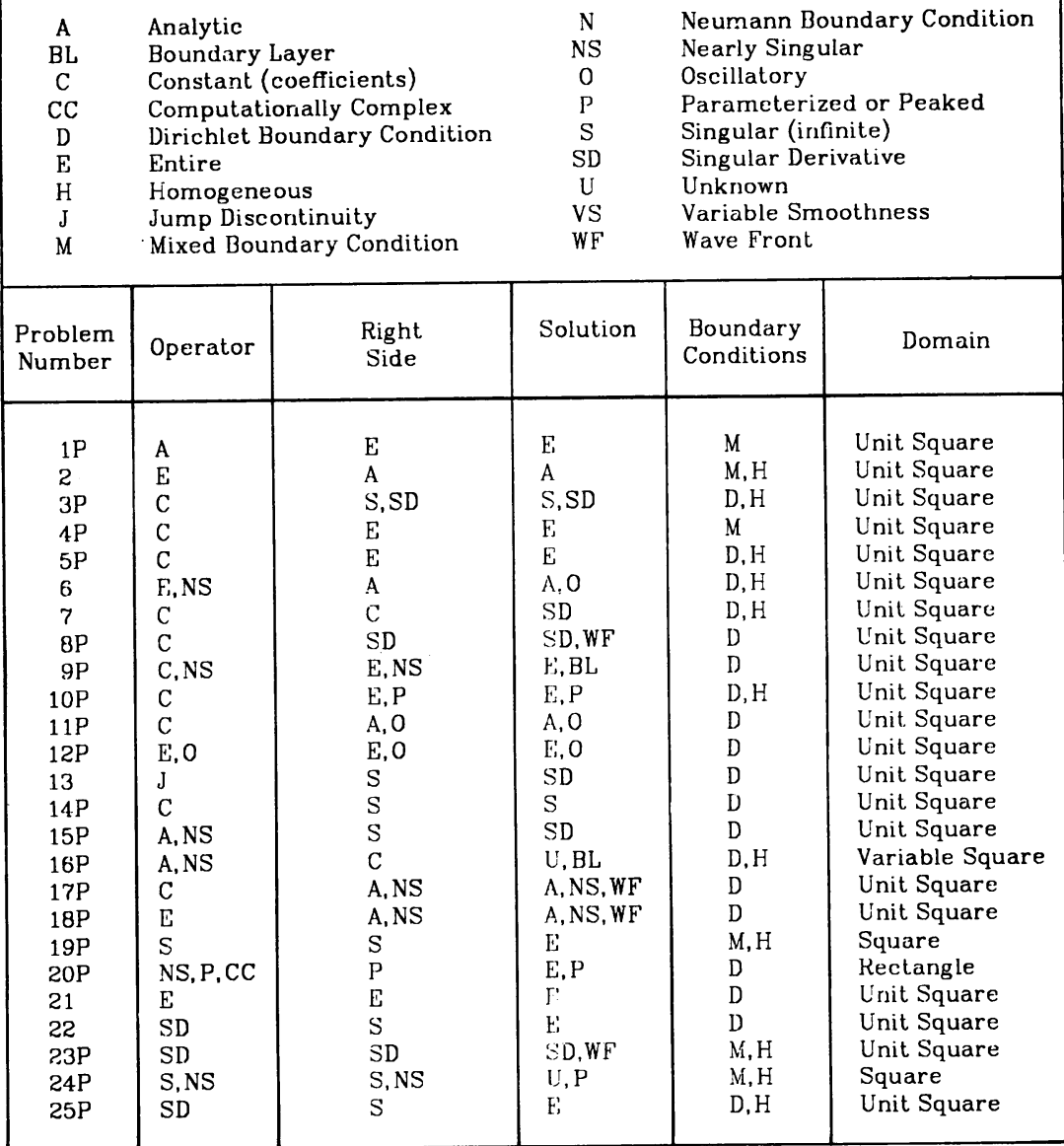




\begin{tabular}{|c|c|c|c|c|c|}
\hline \multicolumn{6}{|c|}{$\begin{array}{c}\text { Table } 2 \\
\text { Problem Characteristics }\end{array}$} \\
\hline $\begin{array}{l}\text { Problem } \\
\text { Number }\end{array}$ & Operator & $\begin{array}{l}\text { Right } \\
\text { Side }\end{array}$ & Solution & $\begin{array}{c}\text { Boundary } \\
\text { Conditions }\end{array}$ & Domain \\
\hline $26 P$ & A & $A$ & U,SD & $\mathrm{D}, \mathrm{H}$ & Variable Square \\
\hline 27 & A, NS & C & $\mathrm{U}, \mathrm{BL}$ & $\mathrm{D}, \mathrm{H}$ & Square \\
\hline $28 \mathrm{P}$ & $\mathrm{J}$ & $\mathrm{C}$ & U,WF' & D, $\mathrm{H}$ & Square \\
\hline $29 \mathrm{P}$ & $S$ & $\mathrm{H}$ & $\mathrm{U}, \mathrm{VS}, \mathrm{BL}$ & $\mathrm{D}$ & Unit Square \\
\hline $30 P$ & $\mathrm{~A}, \mathrm{CC}$ & $\mathrm{A}, \mathrm{CC}$ & A, NS & $\mathrm{D}$ & Unit Square \\
\hline 31 & $\mathrm{C}$ & $\mathrm{C}$ & $\mathrm{E},(\mathrm{SD})$ & M & Square \\
\hline 32 & $\mathrm{~A}$ & $\mathrm{~A}$ & $\mathrm{~F}_{1}$ & l), $\mathrm{H}$ & Rectangle \\
\hline 33 & $\mathrm{C}$ & E & $\mathrm{E}, \mathrm{O}$ & D) & Rectangle \\
\hline 34 & C & $\mathrm{C}$ & $\mathrm{E},(\mathrm{SD})$ & D & Square \\
\hline $35 \mathrm{P}$ & C & $\mathrm{H}$ & $\mathrm{E}, \mathrm{O}, \mathrm{BL}$ & M & Square \\
\hline $36 \mathrm{P}$ & $S$ & $\mathrm{~S}$ & $\mathrm{~A}, \mathrm{BL}$ & D & Unit Square \\
\hline 37 & $\mathrm{E}$ & $\mathrm{E}$ & $\mathrm{E}$ & D & Unit Square \\
\hline $38 \mathrm{P}$ & $\mathrm{C}$ & $\mathrm{H}$ & E,O, VS & D & Rectangle \\
\hline $39 \mathrm{P}$ & $\mathrm{CC}, \mathrm{S}$ & $\mathrm{CC}, \mathrm{S}$ & U. BL & D, C & Unit. Square \\
\hline $40 \mathrm{P}$ & $\mathrm{E}$ & & A & M & Unit Square \\
\hline $41 \mathrm{P}$ & $\mathrm{C}, \mathrm{NS}$ & SD, NS & SD & $\mathrm{D}, \mathrm{H}$ & Square \\
\hline $42 \mathrm{P}$ & $\mathrm{C}$ & $\mathrm{H}$ & $\Lambda, 0$ & $N$ & Variable Rectangle \\
\hline 43 & $\mathrm{C}$ & $\mathrm{H}$ & $\mathrm{E}$ & M & Squáre \\
\hline $44 \mathrm{P}$ & $\mathrm{CC}$ & $\mathrm{CC}$ & $\mathrm{U}, \mathrm{BL}$ & $\mathrm{D}, \mathrm{H}$ & Unit Square \\
\hline $45 \mathrm{P}$ & $\mathrm{C}, \mathrm{NS}$ & $\mathrm{H}$ & $\mathrm{U}, \mathrm{BL}$ & $\mathrm{D}$ & Unit Square \\
\hline $46 \mathrm{P}$ & $\mathrm{C}, \mathrm{NS}$ & $\mathrm{H}$ & $\mathrm{U}, \mathrm{BL}$ & $\mathrm{D}$ & Variable Rectangle \\
\hline $47 \mathrm{P}$ & $\mathrm{C}$ & $\mathrm{S}$ & SD,VS & $\mathrm{D}$ & Unit Square \\
\hline $48 \mathrm{P}$ & $\mathrm{CC}$ & $\mathrm{CC}$ & $\mathrm{U}$ & $\mathrm{U}$ & Unit Square \\
\hline $49 \mathrm{P}$ & $\mathrm{CC}$ & $\mathrm{CC}$ & $\mathrm{U}, \mathrm{SD}, \mathrm{BL}$ & D, C & Unit Square \\
\hline 50 & $\mathrm{C}$ & $\mathrm{H}$ & $\mathrm{F}, \mathrm{O}$ & $\mathrm{D}$ & Rectangle \\
\hline $51 P$ & $\mathrm{~S}$ & $\mathrm{C}$ & $\mathrm{U}, \mathrm{SD}, \mathrm{WF}$ & $\mathrm{M}, \mathrm{H}$ & Unit Square \\
\hline $52 \mathrm{P}$ & $\mathrm{CC}$ & $\mathrm{H}$ & $\mathrm{U}, \mathrm{O}$ & $\mathrm{M}, \mathrm{C}$ & Unit Square \\
\hline $53 \mathrm{P}$ & C. NS & E., 0 & $\mathrm{E}, \mathrm{O}$ & D) & Unii Square \\
\hline $54 \mathrm{P}$ & $\mathrm{E}, \mathrm{CC}$ & $\mathrm{S}, \mathrm{CC}$ & SD,VS & D & Unit Square \\
\hline $55 \mathrm{P}$ & $\mathrm{C}$ & $\mathrm{H}$ & S,VS, BL & M & Rectingle \\
\hline $56 \mathrm{P}$ & $S$ & $\mathrm{CC}$ & $\mathrm{U}, \mathrm{O},(\mathrm{SD})$ & M & Rectangle \\
\hline
\end{tabular}


Table 3

Classifications of Froblems According to Smuothness of the Operator and Right-Side

(A=Analytic: $\mathrm{C}=$ Constants; $\mathrm{CC}=$ Computationally Complicated; $\mathrm{DD}=\mathrm{Discontinuous}$ Derivatives; $\mathrm{E}=$ Entire;

$\mathrm{O}=$ Oscillatory; $\mathrm{P}=$ Peak; $\mathrm{S}=$ Singul:ar)

\begin{tabular}{|ll|l|}
\hline \multicolumn{2}{|c|}{ Smoothness } & \\
Operator & Right-Side & \\
\hline & & \\
C & C & $7,31,34,35,38,42,43,45,46,50,55$ \\
C & E & $4,5,9,10.33,53$ \\
C & A & 11,17 \\
C & DD & $3,8,41$ \\
C & S & $3,14,47$ \\
C & O & $6,11,53$ \\
C & P & 10 \\
E & E & $12,21,37$ \\
E & A & $2,6,18,40$ \\
E & S & 54 \\
A & C & 16,27 \\
A & E & 1 \\
A & A & $26,30,32$ \\
A & S & 15 \\
DD & C & 28 \\
DD & DD & $13,23,25$ \\
DD & S & 22,25 \\
S & C & $29,51,56$ \\
S & S & $19,24,36$ \\
O & A & 6 \\
O & O & 12, \\
C & CC & 17,18 \\
S & CC & 56 \\
CC & C & 52 \\
CC & P & 20 \\
CC & CC & $30,39,44,48,49,54$ \\
& & \\
\hline & & \\
\hline
\end{tabular}

\begin{tabular}{|l|l|}
\hline \multicolumn{2}{|c|}{ Table 4 } \\
\hline \multicolumn{2}{|c|}{ Classifications of Problems } \\
\hline \multicolumn{2}{|c|}{ According to Smoothness of the Solution } \\
\hline \multicolumn{1}{|c|}{ Solution Smoothness } & \multicolumn{1}{c|}{ Problem Numbers } \\
\hline Entire & $1,4,5,9,10,12,19,20,21,22,25$, \\
Analytic & $21,32,33,34,35,37,38,43,50,53$ \\
Singular Derivatives & $3,6,11,17,19,30,36,40,42$ \\
Oscillatory & $6,11,12,33,35,38,42,50,53,56$ \\
Wave Front & $8,17,18,23,28,51$ \\
Discontinuous Derivatives & 8,23 \\
Singular & 54,55 \\
Boundary Layer & $7,9,15,16,27,29,44,45,46,49$ \\
Peaic & $10,20,24$ \\
Tabled Solution & $16,24,26,27,28,29,39,44,45,46$, \\
\hline & $48,49,51,52$ \\
\hline
\end{tabular}

